\title{
A CO-ROTATIONAL FRAMEWORK FOR QUADRILATERAL SHELL ELEMENTS BASED ON THE PURE DEFORMATIONAL METHOD
}

\author{
Y.Q. Tang, Y. P. Liu*, and S. L. Chan \\ Department of Civil and Environmental Engineering, \\ The Hong Kong Polytechnic University, Hong Kong, China; \\ *(Corresponding author: E-mail:yp.liu@polyu.edu.hk)
}

Received: 18 August 2017; Revised: 10 October 2017; Accepted: 19 October 2017

\begin{abstract}
In this paper, a novel co-rotational framework for quadrilateral shell element allowing for the warping effect based on the pure deformational method is proposed for geometrically nonlinear analysis. This new co-rotational framework is essentially an element-independent algorithm which can be combined with any type of quadrilateral shell element. As the pure deformational method is adopted, the quantities of the shell element can be reduced, such that it needs less computer storage and therefore enhances the computational efficiency. In the proposed framework, the geometrical stiffness is derived with a clear physical meaning and regarded as the variations of the nodal internal forces due to the motions and deformations of shell element. Furthermore, the warping effect of quadrilateral shell element is considered, and for this reason, the structures undergoing significant warping can be efficiently solved by the proposed formulation. Finally, several benchmark examples are used to verify the validation and accuracy of the proposed method for geometrically nonlinear analysis.
\end{abstract}

Keywords: element-independence, co-rotational method, flat quadrilateral shell element, pure deformation, geometrically nonlinear analysis, explicit tangent stiffness

DOI: $10.18057 /$ IJASC. 2018.14.1.6

\section{INTRODUCTION}

Shell structures and steel frames made up of thin-wall members are commonly used in civil and structural engineering. Generally, it needs huge computer time to analyze a structure by finite shell elements compared with beam-column elements, especially in nonlinear analyses. Thus, the development of shell elements with high performance as well as the co-rotational framework with improved computational efficiency continuously attracts the attention of many researchers.

There are three common methods based on the Lagrangian kinematic description for geometrically nonlinear analyses, i.e. total Lagrangian (TL), updated Lagrangian (UL) and co-rotational approaches. The last method is latest and attracts more attention than the others recently due to its simplicity and efficiency. The basic idea of the co-rotational method is that the total motions of an element can be divided into two parts, including the rigid body motions and the pure deformations. To decompose them, a local frame is attached to and co-rotated with the element. Then, the translations and rotations of the local frame are taken as the rigid body movements, while the deformations of the element produced in the local frame are regarded as the pure deformations. In reality, the classification of the Lagrangian kinematic descriptions for geometrically nonlinear analysis is not absolute. The co-rotational approach can be incorporated with either the total Lagrangian (TL) or the updated Lagrangian (UL) formulations. In the following, some research works related to the co-rotational description are discussed.

The co-rotational approach was firstly introduced by Wempner [1] and further developed by authors (Belytschko and Hsieh [2]; Belytschko et al. [3]). After almost 50 years' development, 
many versions of co-rotational approaches have been proposed for different kinds of finite elements. The difference of them lies in the treatment of large rotations, simplification of procedure, derivation of geometrical stiffness, definition of local coordinate system, consideration of large strains and so on. The concept of "element-independent co-rotational (EICR) formulation" was first introduced by Rankin and Brogan [4] in 1986. By this formulation, the existing linear finite elements can be easily extended to geometrically nonlinear analysis. Basically, the property of the element-independence comes from that the geometrical stiffness formulated by local coordinates and nodal internal forces at current configuration is explicit, so there is no need to conduct numerical integration over the element. The geometrical stiffness is independent of the local element and therefore any linear element can be extended to geometrically nonlinear analysis with this co-rotational algorithm. Rankin and Brogan [4] presented the EICR algorithm as a general framework, then authors (Rankin and Nour-Omid [5]; Nour-Omid and Rankin [6]) pointed out that the element-independent approach presented in reference (Rankin and Brogan [4]) cannot keep the internal force filed self-equilibrium and further proposed a projector operator to improve its performance. In addition, due to the non-additive nature of large rotations in a three-dimensional space, a pertaining transformation matrix was presented in reference (Nour-Omid and Rankin [6]). The detailed derivation of the EICR method also can be found in references (Crisfield [7]; Felippa and Haugen [8]), in which Felippa and Haugen [8] introduced several related techniques, such as the mathematics of finite rotations, the fitting methods to satisfy invariance to nodal ordering and so on. The pertaining studies and applications for shell elements also can be found in references (Pacoste [9]; Skallerud and Haugen [10]; Eriksson and Pacoste [11]; Battini and Pacoste [12]; Battini and Pacoste [13]). Another similar co-rotational procedure used for a flat triangular shell element was proposed by authors (Levy and his co-workers Levy and Gal [14]; Gal and Levy [15]; Levy and Spillers [16]), in which the derivation of the geometrical stiffness matrix is derived with the load perturbation of the linear equilibrium equations in its local coordinates system.

Crisfield and Moita [17] presented a unified co-rotational framework for solids, shells and beams, which has strong links with the work done by Nour-Omid and Rankin [6] and inherits the merit of element-independence. It can be regarded as a simplified version since some terms are omitted in the derivation of the internal forces and the tangent stiffness. In addition, Crisfield et al. [18] pointed out that the co-rotational technique is restricted in the local element with small strains and can be extended to problems with large strains, in which the local element adopted Biot-stress formulation. Similar to this simpler co-rotational framework, Izzuddin [19] defined a new local co-rotational system and adopted vectorial rotation for quadrilateral shell elements, through which not only the invariance to node ordering but also a symmetric tangent stiffness matrix can be achieved. Pertaining studies on shell elements with this co-rotational framework were also presented in references ( $\mathrm{Li}$ and Vu-Quoc [20]; Li et al. [21]; Li et al. [22]; Li et al. [23]; Li et al. [24]; Izzuddin and Liang [25]; Li et al. [26]).

Also, the co-rotational concept can be integrated with the TL or UL formulation, in which the local tangent stiffness matrix is still obtained through the standard TL or UL formulation described by Bathe [27], while the internal forces are evaluated by the co-rotational approach. For example, based on the geometrically nonlinear flat triangular shell element proposed by Bathe and Ho [28] which is restricted to small rotations between two load steps, Hsiao Kuo-Mo [29] removed the restriction by using the co-rotational approach to eliminate the rigid body motions of the element and get the internal forces. The similar studies of the co-rotational TL or UL formulation for shell elements also can be found in references (Jiang et al. [30]; Hsiao [31]; Tang et al. [32]).

The pure deformational method, also called natural mode method, was firstly proposed by Argyris et al. [33] in the late 1960s, in which the pure deformation modes are separated from the rigid body movements of the local element. This method has substantial computational advantages, since, 
when the rigid body movement is removed, the quantities based on the basic coordinate system are less and the formulation is simpler. The pure deformation method has been widely used in the derivations of beam-column elements. Taking a two-node beam-column element in a plane for example, it has three degrees of freedom (two rotations and one translation) per node and then produces a $6 \times 6$ singular stiffness matrix. However, if using the pure deformational method, the element only has three degrees of freedom (two rotations and one translation) and deduces a $3 \times 3$ non-singular matrix with simpler expression. Thus, the benefits of the pure deformational method are remarkable. Furthermore, it is an element-independent approach and can be applied to many kinds of beam-column elements. For instance, Chan [34] investigated a cubic beam-column element with second-order effect in the large deflection analysis; Chan and Zhou [35] proposed a fifth-order polynomial beam-column element with second-order effect; Tang et al. [36] presented a cubic beam-column element allowing for shear deformation and second order effect simultaneously; similarly, the stability function can be derived through the pure deformational element model as investigated by $\mathrm{Chan}$ and $\mathrm{Gu}$ [37]. The studies of the pure deformational method for shell elements are also useful, but rare, which motivates the authors to explore. And the related work for flat triangular shell elements was also proposed by authors (Tang et al. [38]).

In this paper, a novel pure deformational method for quadrilateral shell elements is proposed. Unlike beam-column elements, the pure deformational formulation becomes more complicated in shell elements. To construct a flat quadrilateral shell element, significant attention should be paid to the warping phenomenon which is taken into accounted in this study. In addition, a novel co-rotational framework for quadrilateral shell elements in the geometrically nonlinear analysis is proposed. This new framework belongs to the EICR algorithm and is consistent with the pioneering work done by authors (Nour-Omid and Rankin [6] and Levy and Gal [14]). Unlike the previous studies, the proposed co-rotational algorithm is on the basis of the proposed pure deformational method. The outline of the paper is arranged in the following. The pure deformational formulation of the flat quadrilateral shell element consisting of a membrane and a plate element is proposed in Section 2. Then, the novel EICR algorithm integrated with the pure deformational method is detailed in Section 3. Finally, Section 4 shows several benchmark problems to verify the accuracy of the proposed co-rotational framework with a typical flat quadrilateral shell element for geometrically nonlinear analysis.

\section{PURE DEFORMATION SHELL ELEMENT MODEL}

The pure deformational element model is constructed on the basic coordinate system and its stiffness matrix should get rid of the restrained degrees of freedom. To assemble the global stiffness matrix, it requires two-step transformations. The first step is the transformation from a basic coordinate system to a local coordinate system, while the second step is the transformation from a local coordinate system to a global coordinate system. The second transformation can follow a standard procedure in the literature or textbooks, while the first transformation is new and will be firstly introduced in this section.

\subsection{Local and Basic Coordinate Systems}

As shown in Figure 1, $X Y Z$ refers to the global coordinate system while $x y z$ is the local coordinate system. In the local coordinate system, the origin is located at node 1 , and the local $x$-axis is aligned to side 1-2 of the shell element, with the local $z$-axis being the normal of the plane 1-2-4. To consider the warping phenomenon, it is convenient to make the nodes 1,2 and 4 coplanar and therefore the warpage happens at the node 3 only. 


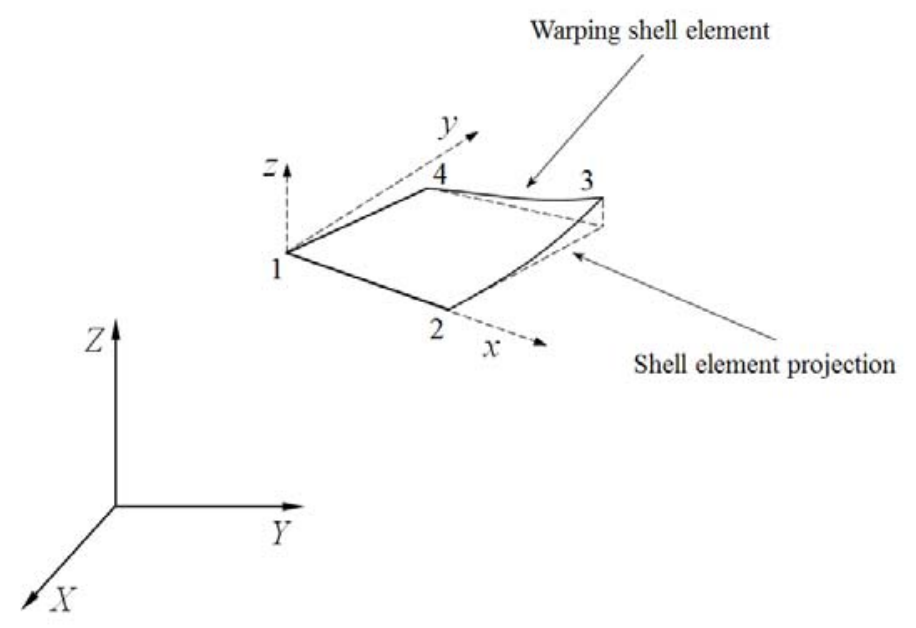

Figure 1. Local Coordinate System

With known the global coordinates of the 4 corner nodes of the shell element, $\boldsymbol{X}_{i}=\left\{X_{i}, Y_{i}, Z_{i}\right\}^{T}$, $i=1,2,3,4$, the three triad vectors of the local coordinate system can be given by,

$\boldsymbol{e}_{x}=\frac{\boldsymbol{X}_{2}-\boldsymbol{X}_{1}}{\left\|\boldsymbol{X}_{2}-\boldsymbol{X}_{1}\right\|}$

$\boldsymbol{e}_{z}=\frac{\boldsymbol{e}_{x} \times\left(\boldsymbol{X}_{4}-\boldsymbol{X}_{1}\right)}{\left\|\boldsymbol{e}_{x} \times\left(\boldsymbol{X}_{4}-\boldsymbol{X}_{1}\right)\right\|}$

$\boldsymbol{e}_{y}=\boldsymbol{e}_{z} \times \boldsymbol{e}_{x}$

and then the transformation matrix from local to global system can be expressed as,

$\boldsymbol{T}=\left[\begin{array}{lll}\boldsymbol{e}_{x} & \boldsymbol{e}_{y} & \boldsymbol{e}_{z}\end{array}\right]^{T}$

The local coordinates of the four corner nodes can be obtained as,

$\boldsymbol{X}_{i}=\boldsymbol{T}\left(\boldsymbol{X}_{i}-\boldsymbol{X}_{1}\right)$

in which $\boldsymbol{x}_{i}=\left\{x_{i}, y_{i}, z_{i}\right\}^{T}, i=1,2,3,4$. Although this definition of the local coordinate system brings nodal ordering dependency, it can be applied to many cases except for some stability problems with symmetry (Battini and Pacoste [12]). More importantly, this definition is convenient to establish the basic coordinate system corresponding to the pure deformational element model.

There is no difference in nodal coordinates between the local and basic coordinate systems. However, the displacement vector and the internal force vector should remove the entries related to rigid body motions when the shell element degenerates from the local coordinate system to the basic one. 
According to Eq. (3) and considering warping phenomenon at the node 3, the nodal coordinates in both the local and basic coordinate systems are given by,

$$
\boldsymbol{x}_{1}=\{0,0,0\}^{T}, \boldsymbol{x}_{2}=\left\{x_{2}, 0,0\right\}^{T}, \boldsymbol{x}_{3}=\left\{x_{3}, y_{3}, z_{3}\right\}^{T}, \boldsymbol{x}_{4}=\left\{x_{4}, y_{4}, 0\right\}^{T}
$$

where the six zero entries in Eq. (4) mean that the corresponding degrees of freedom are restrained in the basic coordinate system.

The displacement vector $\boldsymbol{u}$ and the internal force vector $\boldsymbol{f}$ of the shell element in the local coordinate system are represented by,

$$
\begin{aligned}
& \boldsymbol{u}=\left\{\boldsymbol{u}_{1}^{T}, \boldsymbol{u}_{2}{ }^{T}, \boldsymbol{u}_{3}{ }^{T}, \boldsymbol{u}_{4}{ }^{T}\right\}^{T} \\
& \boldsymbol{f}=\left\{\boldsymbol{f}_{1}{ }^{T}, \boldsymbol{f}_{2}{ }^{T}, \boldsymbol{f}_{3}{ }^{T}, \boldsymbol{f}_{4}{ }^{T}\right\}^{T} \\
& \boldsymbol{u}_{i}=\left\{u x_{i}, u y_{i}, u z_{i}, \theta x_{i}, \theta y_{i}, \theta z_{i}\right\}^{T} \\
& \boldsymbol{f}_{i}=\left\{f x_{i}, f y_{i}, f z_{i}, m x_{i}, m y_{i}, m z_{i}\right\}^{T}
\end{aligned}
$$

For the membrane element with drilling rotation, the displacement vector $\boldsymbol{e}_{m}$ and the corresponding internal force vector $\boldsymbol{p}_{m}$ in the basic coordinate system are defined by,

$$
\begin{aligned}
& \boldsymbol{e}_{m}=\left\{e x_{2}, e x_{3}, e y_{3}, e x_{4}, e y_{4}, \varphi z_{1}, \varphi z_{2}, \varphi z_{3}, \varphi z_{4}\right\}^{T} \\
& \boldsymbol{p}_{m}=\left\{p x_{2}, p x_{3}, p y_{3}, p x_{4}, p y_{4}, n z_{1}, n z_{2}, n z_{3}, n z_{4}\right\}^{T}
\end{aligned}
$$

For the plate element, the displacement vector $\boldsymbol{e}_{p}$ and the corresponding internal force vector $\boldsymbol{p}_{p}$ in the basic coordinate system are given by,

$$
\begin{gathered}
\boldsymbol{e}_{p}=\left\{e z_{3}, \varphi x_{1}, \varphi y_{1}, \varphi x_{2}, \varphi y_{2}, \varphi x_{3}, \varphi y_{3}, \varphi x_{4}, \varphi y_{4}\right\}^{T} \\
\boldsymbol{p}_{p}=\left\{p z_{3}, n x_{1}, n y_{1}, n x_{2}, n y_{2}, n x_{3}, n y_{3}, n x_{4}, n y_{4}\right\}^{T}
\end{gathered}
$$

Note that each vector of the membrane and plate elements has only nine entries. Thus, the displacement vector $\boldsymbol{e}$ and the internal force vector $\boldsymbol{p}$ of the shell element defined in the basic coordinate system can be expressed as follows,

$$
\begin{aligned}
& \boldsymbol{e}=\left\{\boldsymbol{e}_{m}{ }^{T}, \boldsymbol{e}_{p}{ }^{T}\right\}^{T} \\
& \boldsymbol{p}=\left\{\boldsymbol{p}_{m}{ }^{T}, \boldsymbol{p}_{p}{ }^{T}\right\}^{T}
\end{aligned}
$$

\subsection{Transformation between Local and Basic Coordinate Systems}

Focusing on the local coordinate system at first, the shell element has a virtual displacement vector $\delta \boldsymbol{u}$. Then, the variations of rigid body rotations of the element in the local coordinate system can be written as follows, 


$$
\begin{aligned}
& \delta \omega_{x}=\frac{\delta u z_{4}-\delta u z_{1}}{y_{4}}-\frac{\delta u z_{2}-\delta u z_{1}}{y_{4}} \frac{x_{4}}{x_{2}} \\
& \delta \omega_{y}=-\frac{\delta u z_{2}-\delta u z_{1}}{x_{2}} \\
& \delta \omega_{z}=\frac{\delta u y_{2}-\delta u y_{1}}{x_{2}}
\end{aligned}
$$

Thus, as shown in Figure 2, the variations of the pure deformations of the membrane element with boundary conditions can be given by,

$$
\begin{aligned}
& \delta e x_{2}=\delta u x_{2}-\delta u x_{1} \\
& \delta e x_{3}=\delta u x_{3}-\delta u x_{1}+y_{3} \delta \omega_{z}-z_{3} \delta \omega_{y} \\
& \delta e y_{3}=\delta u y_{3}-\delta u y_{1}-x_{3} \delta \omega_{z}+z_{3} \delta \omega_{x} \\
& \delta e x_{4}=\delta u x_{4}-\delta u x_{1}+y_{4} \delta \omega_{z} \\
& \delta e y_{4}=\delta u y_{4}-\delta u y_{1}-x_{4} \delta \omega_{z} \\
& \delta \varphi z_{i}=\delta \theta z_{i}-\delta \omega_{z} \quad(i=1,2,3)
\end{aligned}
$$

in which the warping in the node 3 is taken into account in Eqs. (10b, c).

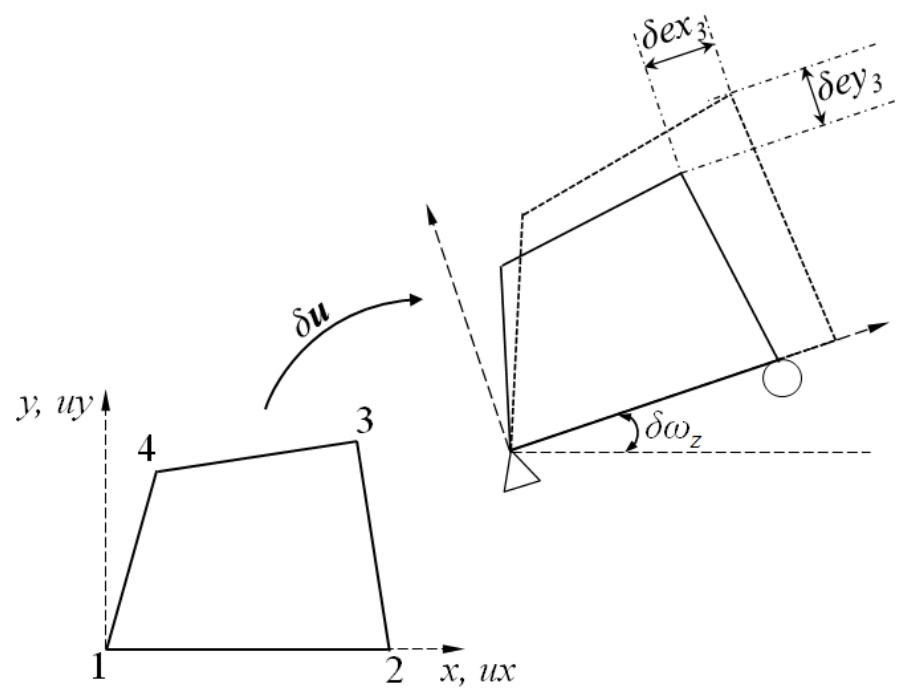

Figure 2. Deformations and Movements of Membrane Element

Then, the transformation matrix for the membrane element from local to basic coordinate system can be derived as, 


$$
\begin{gathered}
\boldsymbol{L}_{m}=\frac{\partial \boldsymbol{e}_{m}}{\partial \boldsymbol{u}^{T}} \\
\delta \boldsymbol{e}_{m}=\boldsymbol{L}_{m} \delta \boldsymbol{u}
\end{gathered}
$$

where $\boldsymbol{L}_{m}$ is a $9 \times 24$ matrix. By the principle of conjugation, the relationship of the internal force vectors between these two different systems can be given by

$$
\boldsymbol{f}=\boldsymbol{L}_{m}{ }^{T} \boldsymbol{p}_{m}
$$

Similarly, as shown in Figure 3, the pure deformations of the plate element with boundary conditions to eliminate rigid body motions of the element can be deduced in the following,

$$
\begin{aligned}
& \delta e z_{3}=\delta u z_{3}-\delta u z_{1}-y_{3} \delta \omega_{x}+x_{3} \delta \omega_{y} \\
& \delta \varphi x_{i}=\delta \theta x_{i}-\delta \omega_{x} \quad(i=1,2,3,4) \\
& \delta \varphi y_{i}=\delta \theta y_{i}-\delta \omega_{y} \quad(i=1,2,3,4)
\end{aligned}
$$

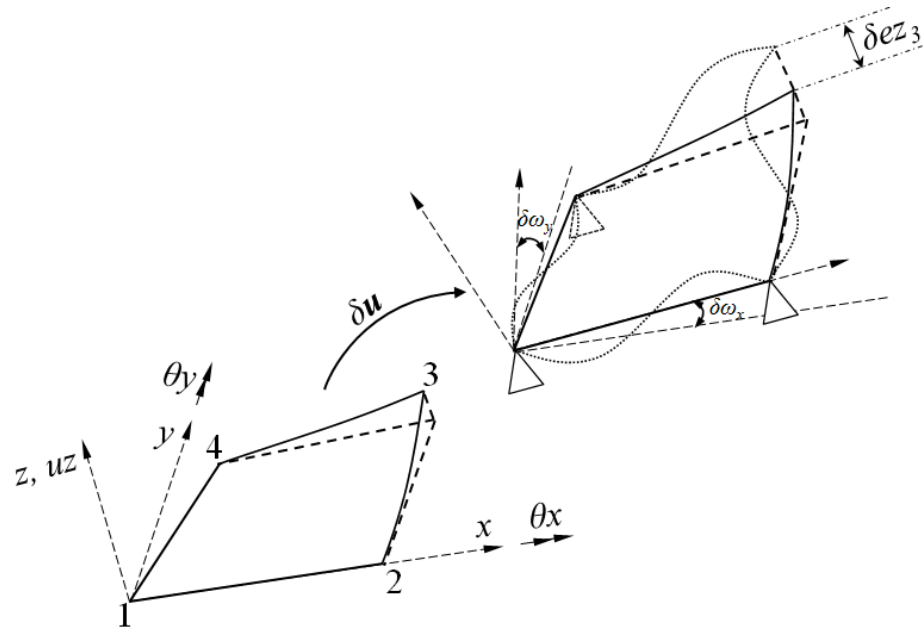

Figure 3. Deformations and Movements of Plate Element

As no out-of-plane restraint is assumed on the node 3 to allow for warping, there exists a vertical displacement at the node 3 given in Eq. (13a). The transformation matrix for the plate element from local to basic coordinate system can be expressed as follows,

$$
\begin{gathered}
\boldsymbol{L}_{p}=\frac{\partial \boldsymbol{e}_{p}}{\partial \boldsymbol{u}^{T}} \\
\delta \boldsymbol{e}_{p}=\boldsymbol{L}_{p} \delta \boldsymbol{u}
\end{gathered}
$$

in which $\boldsymbol{L}_{p}$ is a $9 \times 24$ matrix as well as $\boldsymbol{L}_{m}$. Similar to the membrane element, the internal force vectors of the plate element in these two coordinate systems have the relationship as,

$$
\boldsymbol{f}=\boldsymbol{L}_{p}^{T} \boldsymbol{p}_{p}
$$


Combining the relationships of the membrane and plate elements together gives

$$
\begin{gathered}
\delta \boldsymbol{e}=\boldsymbol{L} \delta \boldsymbol{u} \\
\boldsymbol{f}=\boldsymbol{L}^{T} \boldsymbol{p} \\
\boldsymbol{L}=\left[\begin{array}{l}
\boldsymbol{L}_{m} \\
\boldsymbol{L}_{p}
\end{array}\right]
\end{gathered}
$$

From the above, the relationships of the displacement vectors and the internal force vectors of the shell element between the local and basic coordinate systems have been presented. It is worth noting that the basic coordinate system corresponds to the warping shell element. However, in general, the membrane element and the plate element are derived based on the flat facet which is the projection of the warping shell element. Thus, the transformation between the projection and the warping shell element should be established. In this paper, the warping at the node 3 is regarded as an eccentricity, which can be treated as a rigid beam between the real node and the projected one. According to this assumption, the displacements of the membrane element have the equations as follows,

$$
\begin{aligned}
& \delta e x_{3}^{p}=\delta e x_{3}-z_{3} \delta \varphi y_{3} \\
& \delta e y_{3}^{p}=\delta e y_{3}+z_{3} \delta \varphi x_{3}
\end{aligned}
$$

in which the superscript " $p$ " refers to the projection of the warping shell element.

From Eqs. (16) and (17), the relationship between the projection and the local coordinate system can be written as follows,

$$
\begin{aligned}
& \delta \boldsymbol{e}^{p}=\boldsymbol{E} \boldsymbol{L} \delta \boldsymbol{u} \\
& \boldsymbol{f}=\boldsymbol{L}^{T} \boldsymbol{E}^{T} \boldsymbol{p}^{p} \\
& \boldsymbol{E}_{i, i}=1, i=1 \sim 18 ; \quad \boldsymbol{E}_{2,16}=-z_{3} ; \quad \boldsymbol{E}_{3,15}=z_{3}
\end{aligned}
$$

where $\boldsymbol{E}$ represents the transformation matrix from the warping shell element to its projection. It should be pointed out the plate element is not affected by the warping effect. Thus, the pure deflection and rotations at the node 3 of the warping shell element are identical to those in its projection.

\subsection{Linear Stiffness Matrix in Local Coordinate System}

As shown in Figures 2 and 3, the stiffness matrix of the projection of the warping shell element without rigid body motions is a $18 \times 18$ non-singular matrix and can be expressed as, $\delta \boldsymbol{p}^{p}=\boldsymbol{k}_{b}^{p} \delta \boldsymbol{e}^{p}$

where the subscript " $p$ " means that the stiffness matrix $\boldsymbol{k}_{b}^{p}$ is the basic linear stiffness of the shell element on the projection plane. Because the pure deformational method is element-independent, 
the shell linear stiffness matrix $\boldsymbol{k}_{b}^{p}$ can be formulated by any 4-node membrane element and plate element. Also, the material nonlinearity can be conveniently considered in $\boldsymbol{k}_{b}^{p}$ in the basic coordinate system.

From Eqs. (18) and (19), the stiffness matrix of the warping shell element in the local coordinate system can be derived as follows,

$$
\begin{aligned}
\delta \boldsymbol{f} & =\boldsymbol{L}^{T} \boldsymbol{E}^{T} \boldsymbol{k}_{b}^{p} \boldsymbol{E} \boldsymbol{L} \delta \boldsymbol{u}+\delta\left(\boldsymbol{L}^{T} \boldsymbol{E}^{T}\right) \boldsymbol{p}^{p} \\
& =\boldsymbol{k}_{l} \delta \boldsymbol{u}+\boldsymbol{k}_{g} \delta \boldsymbol{u} \\
\boldsymbol{k}_{l}= & \boldsymbol{L}^{T} \boldsymbol{E}^{T} \boldsymbol{k}_{b}^{p} \boldsymbol{E} \boldsymbol{L}
\end{aligned}
$$

where $\boldsymbol{k}_{l}$ is the stiffness matrix of the warping shell element in the local coordinate system and is a $24 \times 24$ singular stiffness matrix in the local coordinate system. The local geometrical stiffness (initial stress) matrix $\boldsymbol{k}_{g}$ used in geometrically nonlinear analysis will be discussed in the next section.

To sum up, the $18 \times 18$ stiffness matrix $\boldsymbol{k}_{b}^{p}$ has a simpler expression and less dimensions compared with the $24 \times 24$ stiffness matrix $\boldsymbol{k}$, which saves the computer storage and improves the computational efficiency.

\section{CO-ROTATIONAL ALGORITHM}

In this section, a novel co-rotational algorithm for quadrilateral shell elements considering warping effect based on pure deformational method is proposed. Before the derivation of this algorithm, the relationship between the global and basic coordinate systems should be established. Through Eq. (18b), we have known the expression of the internal force vector in the local coordinate system, and therefore the one in the global coordinate system can be written as

$$
\begin{aligned}
& \boldsymbol{F}=\boldsymbol{T}_{8}^{T} \boldsymbol{L}^{T} \boldsymbol{E}^{T} \boldsymbol{p}^{p} \\
& \boldsymbol{F}=\left\{\boldsymbol{F}_{1}^{T}, \boldsymbol{F}_{2}{ }^{T}, \boldsymbol{F}_{3}{ }^{T}, \boldsymbol{F}_{4}{ }^{T}\right\}^{T} \\
& \boldsymbol{F}_{i}=\left\{F X_{i}, F Y_{i}, F Z_{i}, M X_{i}, M Y_{i}, M Z_{i}\right\}^{T}
\end{aligned}
$$

in which the matrix $\boldsymbol{T}_{8}$ is composed of eight matrices $\boldsymbol{T}$ given in Eq. (2) along diagonal.

Similarly, the corresponding displacement vector can be written as,

$$
\begin{aligned}
& \boldsymbol{U}=\left\{\boldsymbol{U}_{1}{ }^{T}, \boldsymbol{U}_{2}{ }^{T}, \boldsymbol{U}_{3}{ }^{T}, \boldsymbol{U}_{4}{ }^{T}\right\}^{T} \\
& \boldsymbol{U}_{i}=\left\{U X_{i}, U Y_{i}, U Z_{i}, \Theta X_{i}, \Theta Y_{i}, \Theta Z_{i}\right\}
\end{aligned}
$$

Thus, the relationships between these different systems by making use of the previous equations can be concluded in Figure 4 as follows, 


\begin{tabular}{|c|c|c|c|c|c|c|}
\hline $\boldsymbol{p}^{p}, \boldsymbol{e}^{p}$ & & $p, e$ & & $f, u$ & & $\boldsymbol{F}, \boldsymbol{U}$ \\
\hline $18 D O F$ & & $18 D O F$ & & $24 D O F$ & & $24 D O F$ \\
\hline & $p=E^{T} p^{p}$ & & $f=L^{T} p$ & & $F=T_{8}^{T} f$ & \\
\hline Projection & 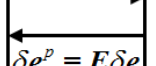 & Basic & \& & Local & 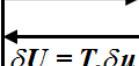 & Total \\
\hline
\end{tabular}

Figure 4. Relationships between Different Coordinate Systems

The conventional co-rotational algorithm needs to consider the non-additive property of 3D large rotations such as references (Nour-Omid and Rankin [6]; Felippa and Haugen [8]) and therefore an additional transformation matrix is required in Eq. (21a). However, this property can be omitted since the pure deformational rotations are small and can be regarded as vectorial parameters. In addition, it can be seen that the warping effect has been taken into account in Eq. (21a), which will contribute to a part of the geometrical stiffness matrix.

According to the derivation process of the EICR algorithm framework presented in references (Nour-Omid and Rankin [6]; Felippa and Haugen [8]) to obtain the tangent stiffness matrix, by taking variation of Eq. (21a), we have,

$$
\delta \boldsymbol{F}=\boldsymbol{T}_{8}^{T} \boldsymbol{L}^{T} \boldsymbol{E}^{T} \delta \boldsymbol{p}^{p}+\delta \boldsymbol{T}_{8}^{T} \boldsymbol{L}^{T} \boldsymbol{E}^{T} \boldsymbol{p}^{p}+\boldsymbol{T}_{8}^{T} \delta \boldsymbol{L}^{T} \boldsymbol{E}^{T} \boldsymbol{p}^{p}+\boldsymbol{T}_{8}^{T} \boldsymbol{L}^{T} \delta \boldsymbol{E}^{T} \boldsymbol{p}^{p}
$$

where the first term yields the linear stiffness matrix and the last three terms produce the geometrical stiffness matrix using the internal forces at current configuration.

Referring to the derivation process by authors (Nour-Omid and Rankin [6]; Felippa and Haugen [8]), it is strict to continuously take variations of these transformation matrices shown in Eq. (22) following standard mathematical flow. However, based on the pure deformational model, a novel and simpler derivation of the EICR algorithm can be proposed, in which the geometrical stiffness matrix is derived by the load perturbation of the linear equilibrium equations in its local coordinates system.

Moreover, Eq. (22) can be rewritten as follows,

$$
\delta \boldsymbol{F}=\boldsymbol{T}_{8}^{T}\left(\boldsymbol{k}_{l}+\boldsymbol{k}_{g}^{r}+\boldsymbol{k}_{g}^{m}+\boldsymbol{k}_{g}^{p}+\boldsymbol{k}_{g}^{w}\right) \boldsymbol{T}_{8} \delta \boldsymbol{U}
$$

in which the subscript " $l$ " means linear stiffness matrix and the subscript " $g$ " means geometrical stiffness matrix; $\boldsymbol{k}_{l}$ is the linear stiffness matrix considering warping effect given in Eq. (20b) and corresponds to the first term in Eq. (22); $\boldsymbol{k}_{g}^{r}$ is due to the rigid body motions of the element and corresponds to the second term in Eq. (22); $\boldsymbol{k}_{g}^{m}$ and $\boldsymbol{k}_{g}^{p}$ are caused by the dimension changes of the membrane and plate elements respectively and reflect the third term in Eq. (22) together; $\boldsymbol{k}_{g}^{w}$ is due to the warping and corresponds to the last terms in Eq. (22). The details of each geometrical stiffness matrix will be introduced as follows.

\subsection{Geometrical Stiffness due to Rigid Body Motions}

On the basis of the local coordinate system, the variation of the internal force vector at node $i$ due to a small rotation is given by Goldstein Goldstein [39] as 
$\delta \boldsymbol{f}_{i}^{f}=\delta \boldsymbol{\omega} \times \boldsymbol{f}_{i}^{f}=-\operatorname{Spin}\left(\boldsymbol{f}_{i}^{f}\right) \delta \boldsymbol{\omega}$

$\delta \boldsymbol{f}_{i}^{m}=\delta \boldsymbol{\omega} \times \boldsymbol{f}_{i}^{m}=-\operatorname{Spin}\left(\boldsymbol{f}_{i}^{m}\right) \delta \boldsymbol{\omega}$

where $\boldsymbol{f}_{i}^{f}=\left\{f x_{i}, f y_{i}, f z_{i}\right\}^{T}$ and $\boldsymbol{f}_{i}^{m}=\left\{m x_{i}, m y_{i}, m z_{i}\right\}^{T}$ are the translational internal force vector and rotational internal moment vector at the node $i$ respectively; $\delta \omega=\left\{\delta \omega_{x}, \delta \omega_{y}, \delta \omega_{z}\right\}^{T}$ is the variation of rigid body rotation vector shown in Eq. (9) defined in the local coordinate system. The operational symbol Spin(·) can transfer a three-dimensional vector into a skew-symmetric spin tensor, for example,

$\operatorname{spin}\left(\boldsymbol{f}_{i}^{f}\right)=\left[\begin{array}{ccc}0 & -f z_{i} & f y_{i} \\ f z_{i} & 0 & -f x_{i} \\ -f y_{i} & f x_{i} & 0\end{array}\right]$

Through Eqs. (5), (9) and (24), we have,

$\delta \boldsymbol{f}=-\boldsymbol{A} \cdot \boldsymbol{G} \delta \mathbf{u}=\boldsymbol{k}_{g}^{r} \delta \mathbf{u}$

$A=\left[\begin{array}{c}\operatorname{Spin}\left(f_{1}^{f}\right) \\ \operatorname{Spin}\left(f_{1}^{m}\right) \\ \mathrm{M} \\ \operatorname{Spin}\left(f_{4}^{f}\right) \\ \operatorname{Spin}\left(f_{4}^{m}\right)\end{array}\right]$

in which the matrix $\boldsymbol{G}$ is used to connect the variation of the rigid body rotation vector to the variation of the nodal displacement vector and can be expressed as follows,

$\boldsymbol{G}=\frac{\partial \boldsymbol{\omega}}{\partial \boldsymbol{u}^{T}}=\left[\begin{array}{llll}\frac{\partial \boldsymbol{\omega}}{\partial \mathbf{u}_{1}^{T}} & \frac{\partial \boldsymbol{\omega}}{\partial \mathbf{u}_{2}^{T}} & \frac{\partial \boldsymbol{\omega}}{\partial \mathbf{u}_{3}^{T}} & \frac{\partial \boldsymbol{\omega}}{\partial \mathbf{u}_{4}^{T}}\end{array}\right]$

Taking use of Eqs. (5a) and (9), the submatrices of $\boldsymbol{G}$ can be detailed as,

$$
\begin{aligned}
& \frac{\partial \boldsymbol{\omega}}{\partial \mathbf{u}_{1}^{T}}=\left[\begin{array}{cccc}
0 & 0 & \left(x_{4}-x_{2}\right) / x_{2} y_{4} & \\
0 & 0 & 1 / x_{2} & \mathbf{0}_{3 \times 3} \\
0 & -1 / x_{2} & 0 &
\end{array}\right] \\
& \frac{\partial \boldsymbol{\omega}}{\partial \boldsymbol{u}_{2}^{T}}=\left[\begin{array}{cccc}
0 & 0 & -x_{4} / x_{2} y_{4} & \\
0 & 0 & -1 / x_{2} & \mathbf{0}_{3 \times 3} \\
0 & 1 / x_{2} & 0 &
\end{array}\right] \\
& \frac{\partial \boldsymbol{\omega}}{\partial \mathbf{u}_{3}^{T}}=\mathbf{0}_{3 \times 6}
\end{aligned}
$$




$$
\frac{\partial \boldsymbol{\omega}}{\partial \boldsymbol{u}_{4}{ }^{T}}=\left[\begin{array}{cccc}
0 & 0 & 1 / y_{4} & \\
0 & 0 & 0 & \mathbf{0}_{3 \times 3} \\
0 & 0 & 0 &
\end{array}\right]
$$

From the above, the geometrical stiffness matrix of the warping shell element due to rigid body rotations in the local coordinate system, $\boldsymbol{k}_{g}^{r}$, has been presented. Note that the internal forces used in Eq. (26b) belong to the warping shell element at current configuration.

\subsection{Geometrical Stiffness due to Dimension Changes}

As the flat quadrilateral shell element consists of a membrane part and a plate part, the corresponding geometrical stiffness matrices will be discussed separately. First, the geometrical stiffness due to the dimension changes of a membrane element will be detailed.

Based on the pure deformational membrane element model with three degrees of freedom restrained (as shown in Figure 5), the internal forces at the free degrees of freedom can be regarded as action forces which will be kept unchanged after deformation. In the meantime, the internal forces at the constraints can be regarded as the reaction forces and will vary after deformation to keep the self-equilibrium of the element. Thus, the gradients of the reaction forces due to the deformation can be used to deduce the geometrical stiffness of the membrane part directly.

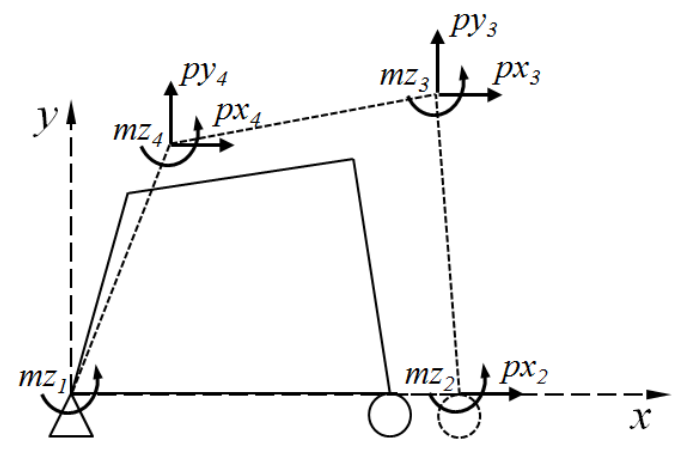

Figure 5. Pure Deformational Membrane Element

Based on the initial configuration of the membrane element, the equilibrium equations can be written as,

$$
\begin{aligned}
& \sum f x=0: f x_{1}+p x_{2}+p x_{3}+p x_{4}=0 \\
& \sum f y=0: f y_{1}+f y_{2}+p y_{3}+p y_{4}=0 \\
& \sum m z=0 \text { at the node } 1: \\
& f y_{2} x_{2}-p x_{3} y_{3}+p y_{3} x_{3}-p x_{4} y_{4}+p y_{4} x_{4}+\sum_{i=1}^{4} n z_{i}=0
\end{aligned}
$$


After deformation, the action forces shown in Figure 5 keep identical, while the three reaction forces $f x_{1}, f y_{1}$ and $f y_{2}$ change to $f x_{1}^{\prime}, f y_{1}^{\prime}$ and $f y_{2}^{\prime}$ respectively. Then, the equilibrium equations become

$$
\begin{aligned}
& \sum f x=0: f x_{1}{ }^{\prime}+p x_{2}+p x_{3}+p x_{4}=0 \\
& \sum f y=0: f y_{1}{ }^{\prime}+f y_{2}{ }^{\prime}+p y_{3}+p y_{4}=0 \\
& \sum m z=0 \text { atthenode } 1: \\
& f y_{2}^{\prime} x_{2}^{\prime}-p x_{3} y_{3}^{\prime}+p y_{3} x_{3}^{\prime}-p x_{4} y_{4}^{\prime}+p y_{4} x_{4}^{\prime}+\sum_{i=1}^{4} n z_{i}=0
\end{aligned}
$$

in which $x_{i}^{\prime}=x_{i}+\delta e x_{i}, i=2,3,4 ; y_{i}^{\prime}=y_{i}+\delta e y_{i}, i=3,4 ; f x_{1}^{\prime}=f x_{1}+\delta f x_{1}, f y_{1}^{\prime}=f y_{1}+\delta f y_{1}$ and $f y_{2}^{\prime}=f y_{2}+\delta f y_{2}$.

Substituting Eq. (29) into Eq. (30) and omitting the high order variations, the variations of the reaction forces yield,

$$
\begin{aligned}
& \delta f x_{1}=0 \\
& \delta f y_{1}=\frac{1}{x_{2}}\left(p x_{3} \delta e y_{3}-p y_{3} \delta e x_{3}+p x_{4} \delta e y_{4}-p y_{4} \delta e x_{4}-f y_{2} \delta e x_{2}\right) \\
& \delta f y_{2}=-\delta f y_{1}
\end{aligned}
$$

Eq. (31) can also be further rewritten as follows,

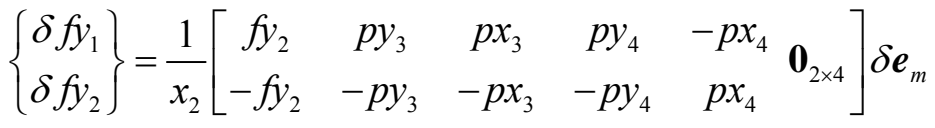

$$
\begin{aligned}
& =\frac{1}{x_{2}}\left[\begin{array}{cccccc}
f y_{2} & p y_{3} & p x_{3} & p y_{4} & -p x_{4} & \mathbf{0}_{2 \times 4} \\
-f y_{2} & -p y_{3} & -p x_{3} & -p y_{4} & p x_{4} &
\end{array}\right] \mathbf{L}_{m} \delta \mathbf{u}_{m} \\
& =\boldsymbol{k}_{g}^{0} \delta \mathbf{u}_{m}
\end{aligned}
$$

in which $k_{g}^{0 / 6}$ is the $2 \times 12$ matrix in the basic coordinate system due to the dimension changes of the membrane element. This matrix needs to be simply expanded to the $24 \times 24$ geometrical stiffness matrix $\boldsymbol{k}_{g}^{m}$ shown in Eq. (23). Also, it should be noted that the warping effect does not influence the equilibrium of the pure deformational membrane element.

Following the same way by finding the variations of the reaction forces, the geometrical stiffness due to the dimension changes of the plate element can be obtained. However, the warping effect should be considered in the plate element. As shown in Figure 6, the nodal vertical displacements are constrained except node 3 . The reaction forces are the shear forces $\left\{f z_{1}, f z_{2}, f z_{4}\right\}^{T}$ at the three restrained nodes. To consider the warping at the node 3 , the in-plane translational forces must be 
taken into account. Unlike the other nodes, the shear force at the node 3 will be kept unchanged after deformation.

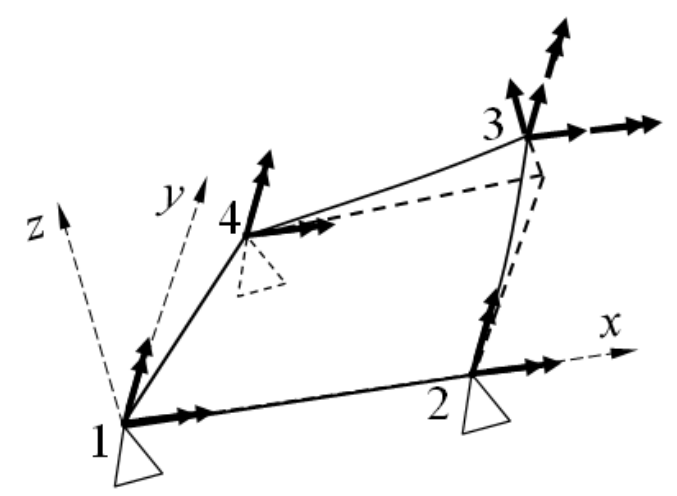

Figure 6. Pure Deformational Plate Element

For the pure deformational plate element at the initial configuration shown in Figure 6, the equilibrium equations can be established as

$$
\begin{aligned}
& \sum f z=0: f z_{1}+f z_{2}+f z_{4}+p z_{3}=0 \\
& \sum m x=0: p z_{3} y_{3}-p y_{3} z_{3}+f z_{4} y_{4}+\sum_{i=1}^{4} n x_{i}=0 \\
& \sum m y=0:-p z_{3} x_{3}+p x_{3} z_{3}-f z_{2} x_{2}-f z_{4} x_{4}+\sum_{i=1}^{4} n y_{i}=0
\end{aligned}
$$

After deformation, the equilibrium equations become

$$
\begin{aligned}
& \sum f z=0: f z_{1}^{\prime}+f z_{2}^{\prime}+f z_{4}^{\prime}+p z_{3}=0 \\
& \sum m x=0: p z_{3} y_{3}^{\prime}-p y_{3} z_{3}^{\prime}+f z_{4}^{\prime} y_{4}^{\prime}+\sum_{i=1}^{4} n x_{i}=0 \\
& \sum m y=0:-p z_{3} x_{3}^{\prime}+p x_{3} z_{3}^{\prime}-f z_{2}^{\prime} x_{2}^{\prime}-f z_{4}^{\prime} x_{4}^{\prime}+\sum_{i=1}^{4} n y_{i}=0
\end{aligned}
$$

in which $x_{i}^{\prime}=x_{i}+\delta e x_{i}, i=2,3,4 ; y_{i}^{\prime}=y_{i}+\delta e y_{i}, i=3,4 ; z_{3}^{\prime}=z_{3}+\delta e z_{3} ; f z_{i}^{\prime}=f z_{i}+\delta f z_{i}, i=1,2,4$.

Considering Eqs. (33) and (34) and omitting the high order variations, we have,

$$
\begin{aligned}
& \delta f z_{4}=\frac{1}{y_{4}}\left(p y_{3} \delta e z_{3}-p z_{3} \delta e y_{3}-f z_{4} \delta e y_{4}\right) \\
& \delta f z_{2}=\frac{1}{x_{2}}\left(-f z_{2} \delta e x_{3}-f z_{4} \delta e x_{4}-p z_{3} \delta e x_{3}+p x \delta e z_{3}-\delta f z_{4} x_{4}\right)
\end{aligned}
$$


$\delta f z_{3}=-\delta f z_{2}-\delta f z_{4}$

Then, it can be rewritten as follows,

$$
\left\{\begin{array}{l}
\delta f z_{1} \\
\delta f z_{2} \\
\delta f z_{3}
\end{array}\right\}=\left[\begin{array}{cccccc}
\frac{f z_{2}}{x_{2}} & \frac{p z_{3}}{x_{2}} & \frac{p z_{3}\left(x_{2}-x_{4}\right)}{x_{2} y_{4}} & \frac{f z_{4}}{x_{2}} & \frac{f z_{4}\left(x_{2}-x_{4}\right)}{x_{2} y_{4}} & \frac{p y_{3}\left(-x_{2}+x_{4}\right)-p x_{3} y_{4}}{x_{2} y_{4}} \\
-\frac{f z_{2}}{x_{2}} & -\frac{p z_{3}}{x_{2}} & \frac{p z_{3} x_{4}}{x_{2} y_{4}} & -\frac{f z_{4}}{x_{2}} & \frac{f z_{4} x_{4}}{x_{2} y_{4}} & \frac{p x_{3} y_{4}-p y_{3} x_{4}}{x_{2} y_{4}} \\
0 & 0 & -\frac{p z_{3}}{y_{4}} & 0 & -\frac{f z_{4}}{y_{4}} & \frac{p y_{3}}{y_{4}}
\end{array}\right]\left\{\begin{array}{l}
\delta e x_{2} \\
\delta e x_{3} \\
\delta e y_{3} \\
\delta e x_{4} \\
\delta e y_{4} \\
\delta e z_{3}
\end{array}\right\}
$$

where $k_{g}^{0 / 3}$ is the $3 \times 12$ geometrical stiffness matrix in the basic coordinate system due to the dimension changes of the plate element and needs to be expanded to the $24 \times 24$ geometrical stiffness matrix $\boldsymbol{k}_{g}^{p}$ shown in Eq. (23).

\subsection{Geometrical Stiffness due to Warping Effect}

The moments on the node allowing for warping varies with the change of eccentricity $(e z 3)$ and therefore the load perturbation will deduce a part of geometrical stiffness. The virtual displacements and rotations at the node 3 of the warping shell element and its projection have the relationship given in Eq. (17). Thus, the corresponding forces and moments in these two different points have the relationship as,

$n x_{3}=z_{3} p y_{3}+n x_{3}^{p}$

$n y_{3}=-z_{3} p x_{3}+n y_{3}^{p}$

Similar to the previous derivations, the forces and moments at the free DOF of the projection can be regarded as the action forces and moments and keep unchanged after deformation. However, the moments at the warping node 3 vary after deformation and their variations can be expressed as,

$$
\begin{aligned}
& \delta n x_{3}=\delta e z_{3} p y_{3} \\
& \delta n y_{3}=-\delta e z_{3} p x_{3}
\end{aligned}
$$

Thus, the variation of the internal force vector of the shell element due to warping phenomenon based on the basic coordinate system can be rewritten through Eq. (38) as,

$\delta \boldsymbol{p}=\boldsymbol{k}_{g}^{0} \delta \boldsymbol{e}$

in which the $\boldsymbol{k}_{g}^{0}$ is a $18 \times 18$ matrix and the non-zero entries $\boldsymbol{k}_{g}^{6}(15,10)=p y_{3}, \boldsymbol{k}_{g}^{06}(16,10)=p x_{3}$. Then, transferring it into the local coordinate system, we have, 
$\boldsymbol{k}_{g}^{w}=\boldsymbol{L}^{T} \boldsymbol{k}_{g}^{0} \boldsymbol{L}$

For now, each part of the tangential stiffness matrix shown in Eq. (23) has been obtained.

\subsection{Determination of Internal Forces}

The key issue in the determination of internal forces of the shell element is how to extract the pure deformations from the total deformations. After that, the internal forces $\boldsymbol{p}^{p}$ at the projection can be computed using the linear stiffness matrix of the shell element and further transferred to the global coordinate system through Eq. (21).

For the iteration step $j$, the incremental nodal displacements and rotations of the shell element in the global coordinate system can be given by,

$$
\begin{aligned}
& \Delta \boldsymbol{U}=\left\{\Delta \boldsymbol{U}_{1}{ }^{T}, \Delta \boldsymbol{U}_{2}{ }^{T}, \Delta \boldsymbol{U}_{3}{ }^{T}, \Delta \boldsymbol{U}_{4}{ }^{T}\right\}^{T} \\
& \Delta \boldsymbol{U}_{i}=\left\{\Delta U X_{i}, \Delta U Y_{i}, \Delta U Z_{i}, \Delta \Theta X_{i}, \Delta \Theta Y_{i}, \Delta \Theta Z_{i}\right\}^{T}
\end{aligned}
$$

The information of the examined shell element at the last iteration step $j-1$ are denoted as: the global and local coordinates ${ }^{j-1} \boldsymbol{X}_{i},{ }^{j-1} \boldsymbol{x}_{i},(i=1,2,3,4)$; the local to global transformation matrix

${ }^{j-1} \boldsymbol{T}$; and the internal forces ${ }^{j-1} \boldsymbol{p}^{p}$ at the projection for pure deformational element. Thus, the next procedure is how to update these data with the known incremental global displacements and rotations shown in Eqs. (40).

The global coordinates of the shell element at the iteration step $j$ can be updated as,

$$
{ }^{j} \boldsymbol{X}_{i}={ }^{j-1} \boldsymbol{X}_{i}+\left\{\Delta U X_{i}, \Delta U Y_{i}, \Delta U Z_{i}\right\}^{T}
$$

Then, the local to global coordinate transformation matrix ${ }^{j} \boldsymbol{T}$ and the local coordinates ${ }^{j} \boldsymbol{x}_{i}$ of the shell element can be computed through Eqs. (2) and (3) respectively. In addition, the incremental translations of the pure deformational shell element considering warping effect can be obtained by using the local coordinates ${ }^{j-1} \boldsymbol{x}_{i}$ and ${ }^{j} \boldsymbol{x}_{i}$ as,

$$
\begin{aligned}
& \Delta e x_{2}={ }^{j} x_{2}-{ }^{j-1} x_{2} \\
& \Delta e x_{3}={ }^{j} x_{3}-{ }^{j-1} x_{3}, \Delta e y_{3}={ }^{j} y_{3}-{ }^{j-1} y_{3}, \Delta e z_{3}={ }^{j} z_{3}-{ }^{j-1} z_{3} \\
& \Delta e x_{4}={ }^{j} x_{4}-{ }^{j-1} x_{4}, \Delta e y_{4}={ }^{j} y_{4}-{ }^{j-1} y_{4}
\end{aligned}
$$

To extract the incremental pure rotations of the shell element from the incremental global rotations with considering the non-vectorial properties of 3D large rotations, the Rodrigues-Cayley rotation tensor is adopted here, which is defined with a rotation angle $\theta$ about an unit pseudo-vector $\boldsymbol{n}=\left\{n_{1}, n_{2}, n_{3}\right\}^{T}$. The rotation matrix can be written as, 


$$
\begin{gathered}
\boldsymbol{R}(\boldsymbol{n}, \theta)=\boldsymbol{I}_{3}+N \sin \theta+\boldsymbol{N}^{2}(1-\cos \theta) \\
\boldsymbol{N}=\boldsymbol{S p i n}(\boldsymbol{n})=\left[\begin{array}{ccc}
0 & -n_{3} & n_{2} \\
n_{3} & 0 & -n_{1} \\
-n_{2} & n_{1} & 0
\end{array}\right]
\end{gathered}
$$

where $\theta=\sqrt{\theta_{x}^{2}+\theta_{y}^{2}+\theta_{z}^{2}}$, and the rotation vector corresponding to the rotation tensor is

$$
\boldsymbol{\theta}=\theta \mathbf{n}=\left\{\begin{array}{c}
\theta x \\
\theta y \\
\theta z
\end{array}\right\}
$$

Through Eq. (43), the incremental rotations $\Delta \Theta_{i}=\left\{\Delta \Theta x_{i}, \Delta \Theta y_{i}, \Delta \Theta z_{i}\right\}^{T}$ at the node $i$ in the global coordinate system can be expressed with the Rodrigues-Cayley rotation tensor as,

$$
\Delta \boldsymbol{R}_{i}=\boldsymbol{R}\left(\boldsymbol{n}_{i}, \Delta \boldsymbol{\Theta}_{i}\right) \quad(i=1,2,3,)
$$

in which

$$
\begin{aligned}
& \boldsymbol{n}=\left\{\Delta \Theta x_{i} / \Delta \Theta_{i}, \Delta \Theta y_{i} / \Delta \Theta_{i}, \Delta \Theta z_{i} / \Delta \Theta_{i}\right\}^{T} \\
& \Delta \Theta_{i}=\sqrt{\Delta \Theta x_{i}^{2}+\Delta \Theta y_{i}^{2}+\Delta \Theta z_{i}^{2}}
\end{aligned}
$$

To remove rigid body rotations of the element, the incremental rotation matrix at the node $i$ for the pure deformational state can be given by

$$
\Delta \boldsymbol{r}_{i}={ }^{j-1} \boldsymbol{T} \Delta \boldsymbol{R}_{i}^{j} \boldsymbol{T}^{T}
$$

Then, the pertaining incremental rotations of the rotation matrix given in Eq. (45) can be deduced by the equation in the following,

$$
\Delta \boldsymbol{\theta}\left(\Delta \boldsymbol{r}_{i}\right)=\left\{\begin{array}{l}
\Delta \theta x_{i} \\
\Delta \theta y_{i} \\
\Delta \theta z_{i}
\end{array}\right\}=\frac{\Delta \theta}{2 \sin \Delta \theta}\left\{\begin{array}{l}
\Delta r_{i}^{32}-\Delta r_{i}^{23} \\
\Delta r_{i}^{13}-\Delta r_{i}^{31} \\
\Delta r_{i}^{21}-\Delta r_{i}^{12}
\end{array}\right\}=\frac{\arcsin \Delta \tau}{2 \Delta \tau}\left\{\begin{array}{l}
\Delta r_{i}^{32}-\Delta r_{i}^{23} \\
\Delta r_{i}^{13}-\Delta r_{i}^{31} \\
\Delta r_{i}^{21}-\Delta r_{i}^{12}
\end{array}\right\}
$$

in which, $\Delta \tau=\sqrt{\left(\Delta r_{i}^{32}-\Delta r_{i}^{23}\right)^{2}+\left(\Delta r_{i}^{13}-\Delta r_{i}^{31}\right)^{2}+\left(\Delta r_{i}^{21}-\Delta r_{i}^{12}\right)^{2}}$ and $\Delta r_{i}^{p q} \quad(p, q=1,2,3)$ is the entry of rotation matrix $\Delta \boldsymbol{r}_{i}$.

Note that the range of $\Delta \theta$ in Eq. (46a) is $[0,90]$ and the numerical instability would occur when $\Delta \theta$ is closed to zero. Thus, the Taylor series of Eq. (50a) is adopted when $\theta$ is close to 0 . For $\Delta \theta \leq 0.05$, the coefficient in Eq. (46a) becomes 
$\frac{\Delta \theta}{2 \sin \Delta \theta} \approx \frac{1}{2}+\frac{1}{12} \Delta \theta^{2}+\frac{7}{720} \Delta \theta^{4}$

Thus, by taking advantage of Eq. (46), we can get the pure deformational rotations of the warping shell element in the local coordinate system. As the rotations in the basic coordinate system are identical to those in the local coordinate system, we have,

$\Delta \varphi x_{i}=\Delta \theta x_{i}, \Delta \varphi y_{i}=\Delta \theta y_{i}, \Delta \varphi z_{i}=\Delta \theta z_{i} \quad(i=1,2,3)$

From the above, the 18 entries of the incremental displacement vector $\Delta \boldsymbol{e}$ in the basic coordinate system can be obtained and then transferred to the projection through Eq. (17) as,

$\Delta \boldsymbol{e}^{p}={ }^{j} \boldsymbol{E} \Delta \boldsymbol{e}$

where the matrix ${ }^{j} \boldsymbol{E}$ is calculated by using the local coordinates at current configuration.

Further, the incremental internal force vector of the projection can be determined through Eq. (19) as,

$\Delta \boldsymbol{p}^{p}={ }^{0} \boldsymbol{k}_{b}^{p} \Delta \boldsymbol{e}^{p}$

in which ${ }^{0} \boldsymbol{k}_{b}^{p}$ is the initial stiffness matrix of the shell element at the projection calculated on the basis of initial configuration and used all the time. Thus, this matrix can be stored at the beginning of geometrically nonlinear analysis for all elastic problems. It is clear that this $18 \times 18$ stiffness matrix based on the pure deformation element model needs only $57 \%$ storage of the conventional $24 \times 24$ stiffness matrix.

Then, the internal force vector of the projection at the iteration step $j$ can be updated by,

$$
{ }^{j} \boldsymbol{p}^{p}={ }^{j-1} \boldsymbol{p}^{p}+\Delta \boldsymbol{p}^{p}
$$

Finally, the internal force vector of the warping shell element in the global coordinate system at the current time can be obtained through Eq. (21). It should be noted that the procedures mentioned above imply that the pure deformational rotations are vectorial and additive according to the assumption of small rotations satisfy the classical plate theory.

\section{NUMERICAL EXAMPLES}

In this section, several benchmark examples are presented to illustrate the accuracy and robustness of the proposed EICR formulation based on the pure deformation method. In this study, a quadrilateral shell element formed by the membrane element with drilling rotations proposed by Ibrahimbegovic et al. [40] and the DKQ plate element proposed by Batoz and Tahar [41] is adopted for demonstration. The shell element was also extended into the geometrically nonlinear analysis by authors (Tang et al. [32]; Tang et al. [42]) and obtained success. In fact, any membrane and plate element can be employed in the proposed co-rotational framework. For example, to account for transverse shear deformation, the RDKQM plate element proposed by Chen and Cheung [43] can be used instead of the DKQ element. 
In all examples, the 8-point two-dimensional integration is adopted. The convergent criterion is taken the norm of unbalanced forces not greater than $0.1 \%$ of the norm of the applied load.

\subsection{Cantilever Plate Subjected to End Moment}

A cantilever plate subjected to a concentrated end moment $M$ at its tip is shown in Figure 7 . It is a classical problem for examination of the performance of an element undergoing large rotations. In this example, there is no membrane stress and the cantilever beam will roll up into a complete circle when $M=2 \pi E I / L$. The input data are given in Figure 7.

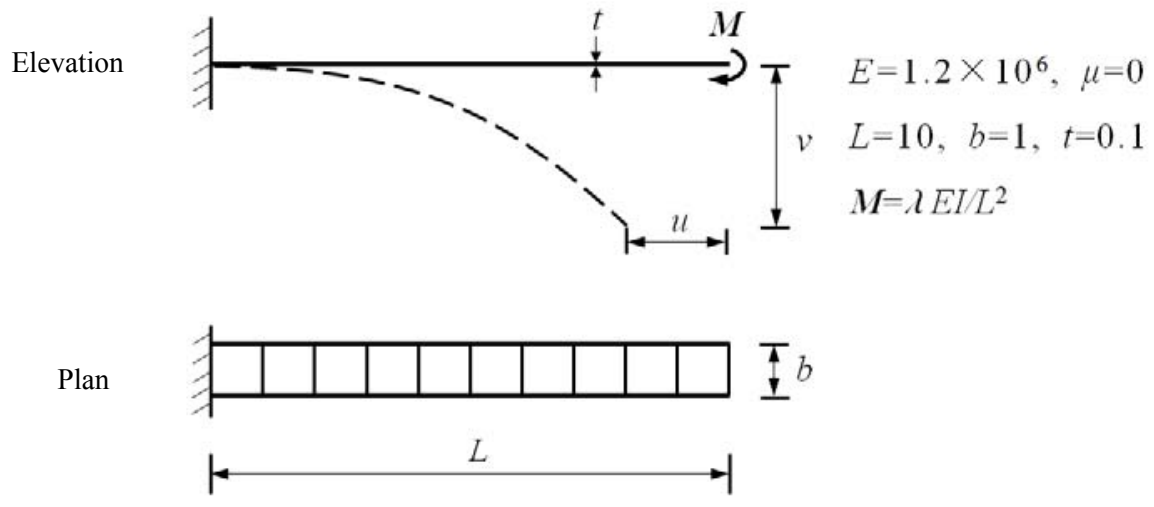

Figure 7. Cantilever Plate Subjected to End Moment

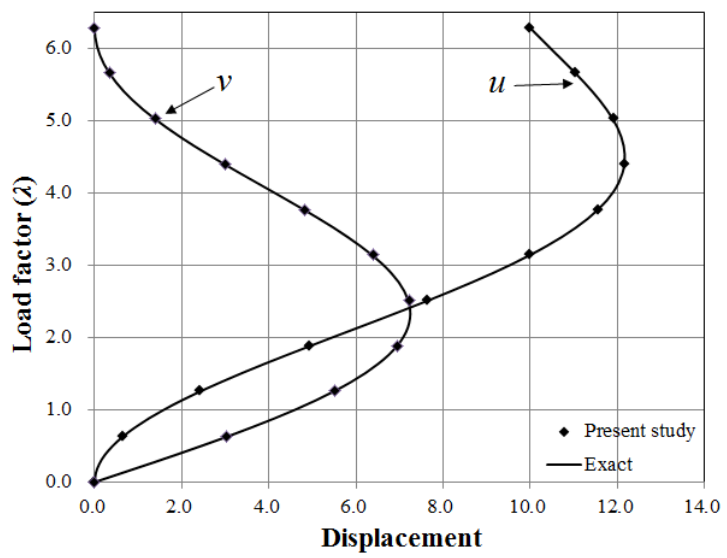

Figure 8. Load-displacement Curves of Cantilever Plate Subjected to An End Moment

The cantilever plate is divided into 10 quadrilateral flat shell elements. The load-displacement curves at the free end are plotted in Figure 8 which shows that these points have a very good agreement with the exact solutions.

\subsection{Cantilever Beam Subjected to Shear Forces}

A cantilever beam is subjected to two equal point loads at the free ends as shown in Figure 9. This example aims to verify the capability of the proposed co-rotational algorithm undergoing large deflections. The configuration and the material of the structure are the same as the first example except for the applied loading and Poisson's ratio $\mu$. 


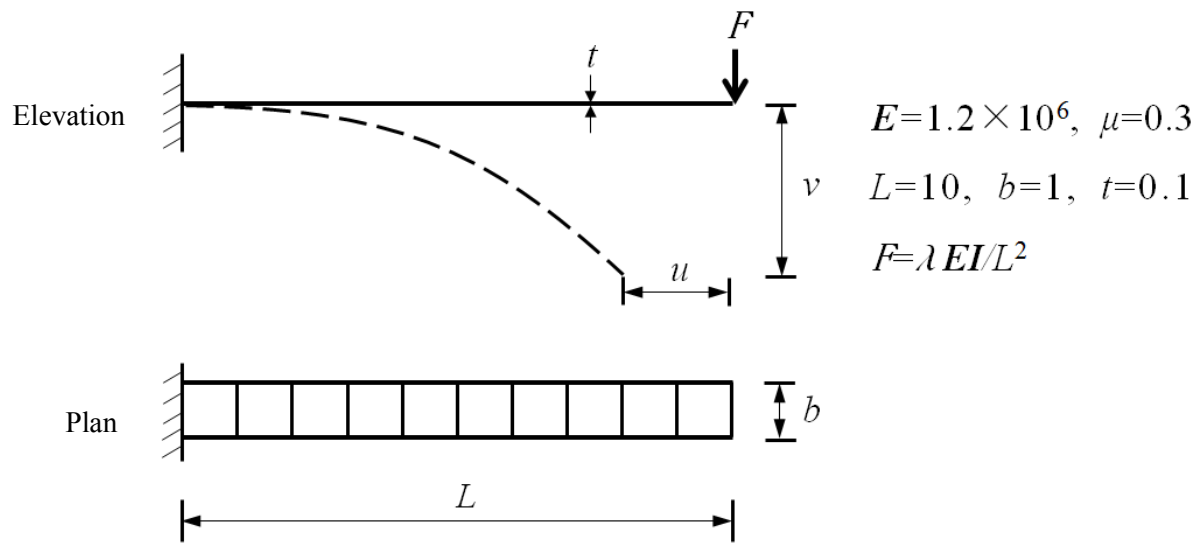

Figure 9. Cantilever Plate Subjected to Shear Forces

The predicted load-displacement curves are plotted against the results using 10 beam-column elements proposed by Tang et al. [36] in Figure 10. It can be seen that the results from the present study agree well with those by Tang et al. [36].

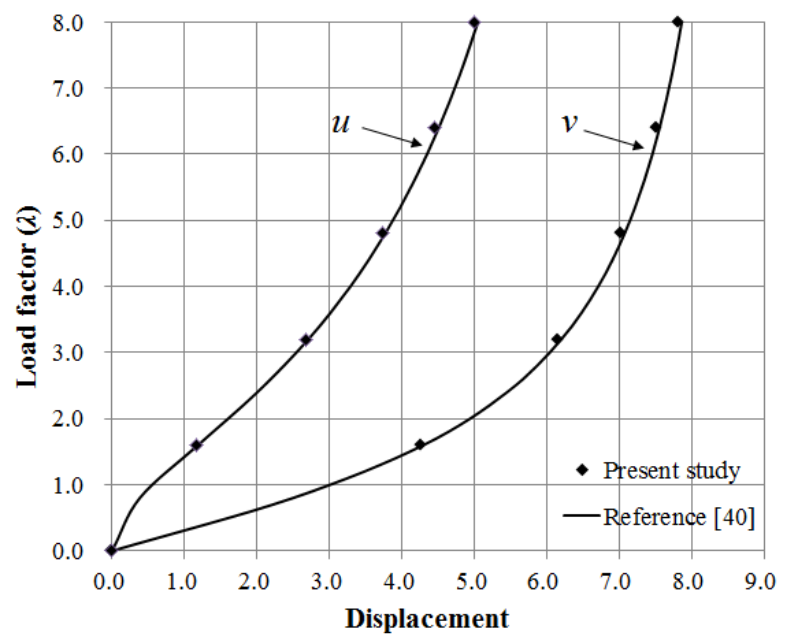

Figure 10. Load-displacement Curves of Cantilever Plate Subjected to Shear Forces

\subsection{Pinched Hemispherical Shell with A Hole}

The studied example is a hemispherical shell with an $18^{\circ}$ hole at the top, as seen in Figure 11. This shell structure is subjected to two inward and two outward forces at the quarter points of its open edge. In this example, severely warping effect occurs for quadrilateral flat shell elements. Due to the symmetry of this problem, only a quarter of the hemisphere uses a $16 \times 16$ mesh of the proposed shell element. 

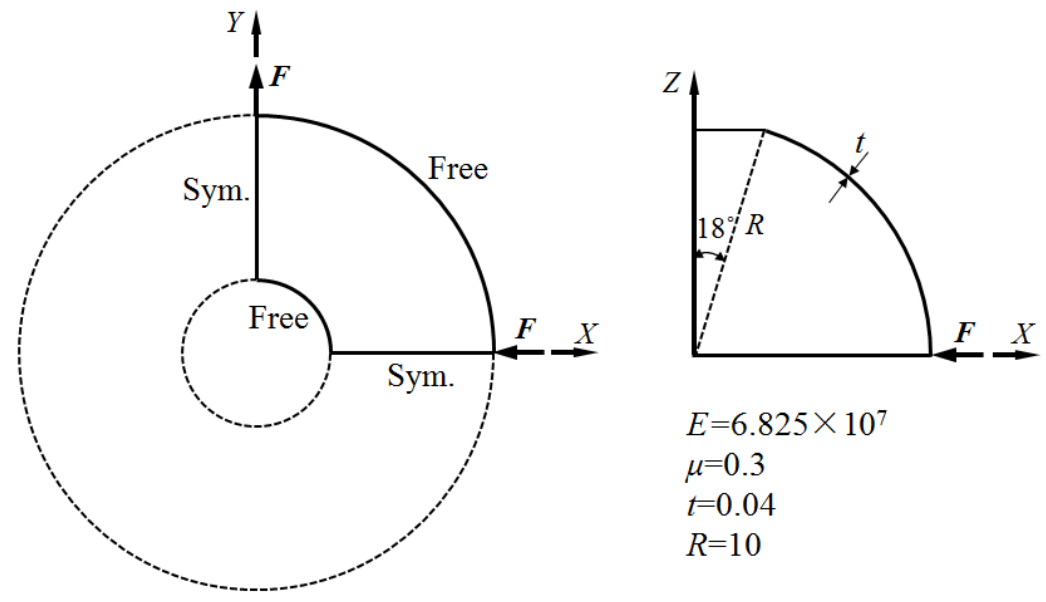

$E=6.825 \times 10^{7}$

$\mu=0.3$

$t=0.04$

$R=10$

Figure 11. Pinched Hemispherical Shell with A Hole

The load-displacement curves at the quarter points obtained by the flat quadrilateral shell element based on the novel EICR formulation are plotted in Figure 12, while the results from the models by $16 \times 16$ Shell 181 elements in ANSYS and $12 \times 12$ MITC3+ (Jeon et al. [44]) elements are also provided for comparison. In general, the present study has the same trends as the others. The proposed method is slightly stiffer than the Shell 181 but softer than the MITC3+ element.

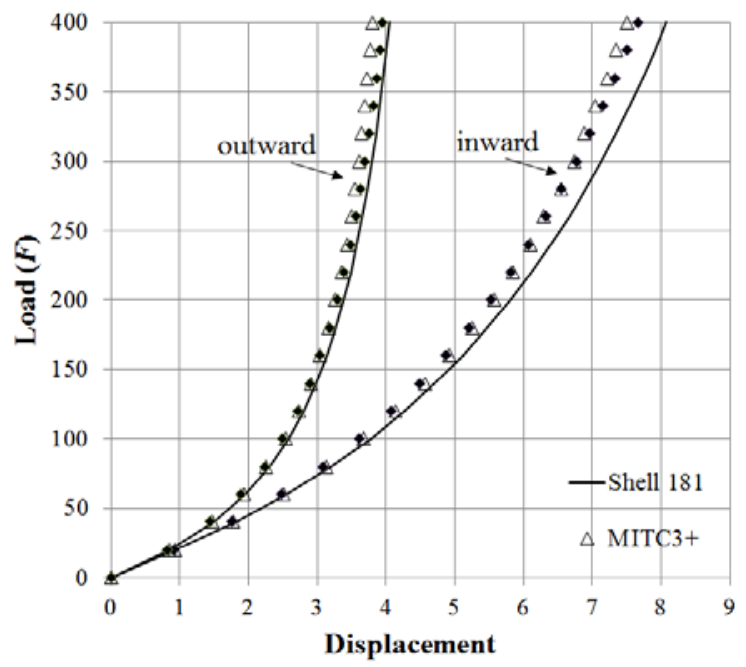

Figure 12. Load-displacement Curves of Pinched Hemispherical Shell

\subsection{Cylindrical Shell Segment}

A shallow cylinder shell is pinned along its longitudinal edges and free at its curved edges, with a concentrated load at its centre as shown in Figure 12. It is a well-known problem used to check the ability of an element against instability behaviour. Its equilibrium path exhibits a snap-through response with two limit points. Following the previous studies, two different thicknesses of 6.35 and 12.7 are considered respectively in this example. The detailed dimensions and material properties are shown in Figure 12. Due to symmetric nature, only a quarter of the structure is meshed with $4 \times 4$ shell elements. In addition, the arc-length method is adopted to trace the full equilibrium path of this problem. 
The predicted results are plotted in Figure 13 against those of the shell 181 element in ANSYS and Surana [45]. It can be seen that the proposed algorithm shows a good agreement with the other elements.

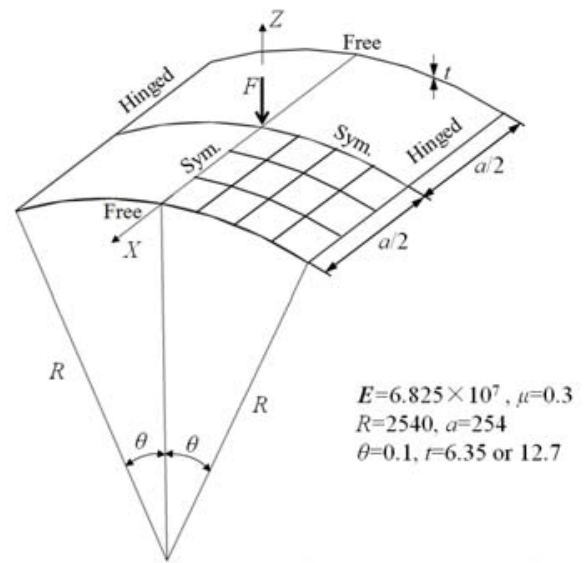

Figure 13. Cylindrical Shell Segment

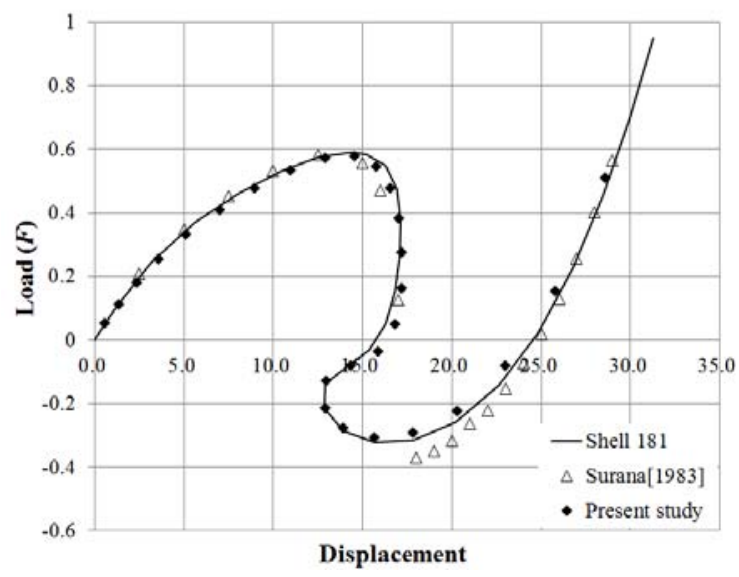

(a) $\mathrm{t}=6.35$

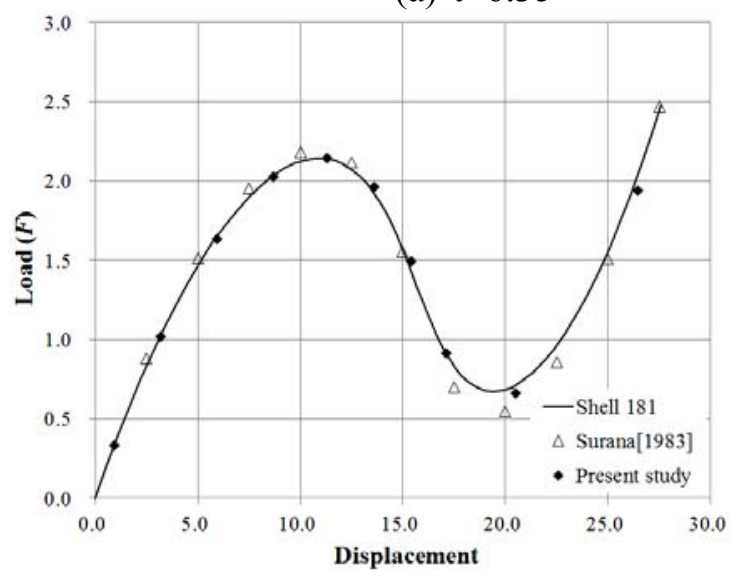

(b) $\mathrm{t}=12.7$

Figure 14. Load-displacement Curves of Pinched Hemispherical Shell 


\section{CONCLUSION}

In this paper, a novel co-rotational algorithm for quadrilateral shell element allowing for warping phenomenon based on pure deformational method is proposed for geometrically nonlinear analysis. This new co-rotational framework is essentially an element-independent algorithm which can be applied to any type of quadrilateral shell element constructed by different displacement interpolations. As the quantities of shell element are reduced by the pure deformational method, it leads to less computer storage and enhances the computational efficiency. Also, the co-rotational framework can consider the warping effect and therefore has a good convergence rate when warping occurs. The numerical examples presented in this paper demonstrate that the proposed method is accurate and robust. From this study, many previous works related to linear 4-node quadrilateral shell element can be conveniently extended to geometrically nonlinear analysis based on the proposed co-rotational framework.

\section{ACKNOWLEDGEMENT}

The authors are grateful to the financial supports by the Research Grant Council of the Hong Kong SAR Government on the projects "Second-Order Analysis of Flexible Steel Cable Nets Supporting Debris (PolyU 152008/15E)" and "Second-order and Advanced Analysis of Arches and Curved Structures (PolyU 152012/14E)", the Hong Kong Branch of Chinese National Engineering Research Centre for Steel Construction supported by The Innovation and Technology Fund of the Hong Kong SAR Government for the project "Advanced Numerical Analyses for Building Structures Using High Performance Steel Materials" and by the Innovative Technology Fund for the project "Advanced design of flexible barrier systems by large deflection theory (ITS/032/14)".

\section{REFERENCES}

[1] Wempner, G., "Finite Elements, Finite Rotations and Small Strains of Flexible Shells", International Journal of Solids and Structures, 1969, Vol. 5, No. 2, pp. 117-153.

[2] Belytschko, T. and Hsieh, B., "Non-linear Transient Finite Element Analysis with Convected Co-ordinates", International Journal for Numerical Methods in Engineering, 1973, Vol. 7, No. 3, pp. 255-271.

[3] Belytschko, T., Schwer, L. and Klein, M., "Large Displacement, Transient Analysis of Space Frames", International Journal for Numerical Methods in Engineering, 1977, Vol. 11, No. 1, pp. 65-84.

[4] Rankin, C. C. and Brogan, F. A., "An Element Independent Corotational Procedure for the Treatment of Large Rotations", Journal of pressure vessel technology, 1986, Vol. 108, No. pp. 165-174.

[5] Rankin, C. and Nour-Omid, B., "The Use of Projectors to Improve Finite Element Performance", Computers \& Structures, 1988, Vol. 30, No. 1, pp. 257-267.

[6] Nour-Omid, B. and Rankin, C., "Finite Rotation Analysis and Consistent Linearization Using Projectors", Computer Methods in Applied Mechanics and Engineering, 1991, Vol. 93, No. 3, pp. 353-384.

[7] Crisfield, M., "Advanced Topics, Volume 2, Non-Linear Finite Element Analysis of Solids and Structures", Wiley, 1997.

[8] Felippa, C. and Haugen, B., "A Unified Formulation of Small-strain Corotational Finite Elements: I. Theory", Computer Methods in Applied Mechanics and Engineering, 2005, Vol. 194, No. 21, pp. 2285-2335. 
[9] Pacoste, C., "Co-rotational Flat Facet Triangular Elements for Shell Instability Analyses", Computer Methods in Applied Mechanics and Engineering, 1998, Vol. 156, No. 1, pp. 75-110.

[10] Skallerud, B. and Haugen, B., "Collapse of Thin Shell Structures-stress Resultant Plasticity Modelling within a Co-rotated ANDES Finite Element Formulation", International Journal for Numerical Methods in Engineering, 1999, Vol. 46, No. 12, pp. 1961-1986.

[11] Eriksson, A. and Pacoste, C., "Element Formulation and Numerical Techniques for Stability Problems in Shells", Computer Methods in Applied Mechanics and Engineering, 2002, Vol. 191, No. 35, pp. 3775-3810.

[12] Battini, J. M. and Pacoste, C., "On the Choice of Local Element Frame for Corotational Triangular Shell Elements", Communications in Numerical Methods in Engineering, 2004, Vol. 20, No. 10, pp. 819-825.

[13] Battini, J. M. and Pacoste, C., "On the Choice of the Linear Element for Corotational Triangular Shells", Computer Methods in Applied Mechanics and Engineering, 2006, Vol. 195, No. 44, pp. 6362-6377.

[14] Levy, R. and Gal, E., "Geometrically Nonlinear Three-noded Flat Triangular Shell Elements", Computers \& Structures, 2001, Vol. 79, No. 26, pp. 2349-2355.

[15] Gal, E. and Levy, R., "Geometrically Nonlinear Analysis of Shell Structures using a Flat Triangular Shell Finite Element", Archives of Computational Methods in Engineering, 2006, Vol. 13, No. 3, pp. 331-388.

[16] Levy, R. and Spillers, W. R. "Analysis of Geometrically Nonlinear Structures", Springer Science \& Business Media, 2013.

[17] Crisfield, M. and Moita, G., "A Unified Co-rotational Framework for Solids, Shells and Beams", International Journal of Solids and Structures, 1996, Vol. 33, No. 20, pp. 2969-2992.

[18] Crisfield, M., Moita, G., Lyons, L. and Jelenić, G., "Enhanced Lower-order Element Formulations for Large Strains", Computational mechanics, 1995, Vol. 17, No. 1-2, pp. 62-73.

[19] Izzuddin, B., "An Enhanced Co-rotational Approach for Large Displacement Analysis of Plates", International Journal for Numerical Methods in Engineering, 2005, Vol. 64, No. 10, pp. 1350-1374.

[20] Li, Z. and Vu-Quoc, L., "An Efficient Co-rotational Formulation for Curved Triangular Shell Element", International Journal for Numerical Methods in Engineering, 2007, Vol. 72, No. 9, pp. 1029-1062.

[21] Li, Z., Izzuddin, B. and Vu-Quoc, L., "A 9-node Co-rotational Quadrilateral Shell Element", Computational Mechanics, 2008, Vol. 42, No. 6, pp. 873-884.

[22] Li, Z., Liu, Y., Izzuddin, B. and Vu-Quoc, L., "A Stabilized Co-rotational Curved Quadrilateral Composite Shell Element", International Journal for Numerical Methods in Engineering, 2011, Vol. 86, No. 8, pp. 975-999.

[23] Li, Z., Zhuo, X., Vu-Quoc, L., Izzuddin, B. and Wei, H., "A Four-node Corotational Quadrilateral Elastoplastic Shell Element using Vectorial Rotational Variables", International Journal for Numerical Methods in Engineering, 2013, Vol. 95, No. 3, pp. 181-211.

[24] Li, Z., Zheng, T., Vu-Quoc, L. and Izzuddin, B., "A 4-node Co-rotational Quadrilateral Composite Shell Element", International Journal of Structural Stability and Dynamics, 2016, Vol. 16, No. 9, Vol. 1550053.

[25] Izzuddin, B. and Liang, Y., "Bisector and Zero-macrospin Co-rotational Systems for Shell Elements", International Journal for Numerical Methods in Engineering, 2016, Vol. 105, No. 4, pp. 286-320.

[26] Li, Z., Izzuddin, B., Vu-Quoc, L., Rong Z. and Zhuo X.. "A 3-node Co-rotational Triangular Elasto-plastic Shell Element using Vectorial Rotational Variables." Advanced Steel Construction, 2017, Vol. 13, No. 3, pp. 206-240.

[27] Bathe, K. J. "Finite Element Procedures", Klaus-Jurgen Bathe, 2006. 
[28] Bathe, K. J. and Ho, L. W., "A Simple and Effective Element for Analysis of General Shell Structures", Computers \& Structures, 1981, Vol. 13, No. 5-6, pp. 673-681.

[29] Kuo, M, H., "Nonlinear Analysis of General Shell Structures by Flat Triangular Shell Element", Computers \& structures, 1987, Vol. 25, No. 5, pp. 665-675.

[30] Jiang, L., Chernuka, M. W. and Pegg, N. G., "A Co-rotational, Updated Lagrangian Formulation for Geometrically Nonlinear Finite Element Analysis of Shell Structures", Finite Elements in Analysis and design, 1994, Vol. 18, No. 1-3, pp. 129-140.

[31] Hsiao, K. M., "Nonlinear Analysis of General Shell Structures by Flat Triangular Shell Element", Computers \& structures, 1987, Vol. 25, No. 5, pp. 665-675.

[32] Tang, Y. Q., Zhou, Z. H. and Chan, S. L., "Geometrically Nonlinear Analysis of Shells by Quadrilateral Flat Shell Element with Drill, Shear and Warping", International Journal for Numerical Methods in Engineering, 2016, Vol. 108, No. 10, pp.1248-1272

[33] Argyris, J. H., Balmer, H., Doltsinis, J. S., Dunne, P. C., Haase, M., Kleiber, M., Malejannakis, G. A., Mlejnek, H. P., Müller, M. and Scharpf, D. W., "Finite Element Method the Natural Approach", Computer Methods in Applied Mechanics and Engineering, 1979, Vol. 17, pp. 1-106.

[34] Chan, S. L., "Large Deflection Kinematic Formulations for Three-dimensional Framed Structures", Computer Methods in Applied Mechanics and Engineering, 1992, Vol. 95, No. 1, pp. 17-36.

[35] Chan, S. L. and Zhou, Z. H., "Pointwise Equilibrating Polynomial Element for Nonlinear Analysis of Frames", Journal of Structural Engineering, 1994, Vol. 120, No. 6, pp. 1703-1717.

[36] Tang, Y. Q., Zhou, Z. H. and Chan, S. L., "Nonlinear Beam-column Element under Consistent Deformation", International Journal of Structural Stability and Dynamics, 2015, Vol. 15, No. 5, pp. 1450068.

[37] Chan, S. L. and Gu, J. X., "Exact Tangent Stiffness for Imperfect Beam-column Members", Journal of Structural Engineering, 2000, Vol. 126, No. 9, pp. 1094-1102.

[38] Tang, Y. Q., Y. P. Liu, and S. L. Chan. "Element-independent Pure Deformational and Co-rotational Methods for Triangular Shell Elements in Geometrically Nonlinear Analysis", International Journal of Structural Stability and Dynamics, 2017, Vol. 18, No. 5, pp. 1850065.

[39] Goldstein, H., "Classical Mechanics", Mass: Addison-Wesley, 1950

[40] Ibrahimbegovic, A., Taylor, R. L. and Wilson, E. L., "A Robust Quadrilateral Membrane Finite Element with Drilling Degrees of Freedom", International Journal for Numerical Methods in Engineering, 1990, Vol. 112, No. 11, pp. 1519-1538.

[41] Batoz, J. L. and Tahar, M. B., "Evaluation of a New Quadrilateral Thin Plate Bending Element", International Journal for Numerical Methods in Engineering, 1982, Vol. 18, No. 11, pp. 1655-1677.

[42] Tang, Y. Q., Zhou, Z. H. and Chan, S. L., "A Simplified Co-rotational Method for Quadrilateral Shell Elements in Geometrically Nonlinear Analysis", International Journal for Numerical Methods in Engineering, 2017, Vol. 15, No. 5, pp. 1450068.

[43] Chen, W. J. and Cheung, Y., "Refined Quadrilateral Element based on Mindlin/Reissner Plate Theory", Int. J. Numer. Meth. Eng., 2000, Vol. 47, No. 1-3, pp. 605-627.

[44] Jeon, H.M., Lee, Y., Lee, P. S. and Bathe, K. J., "The MITC3+ Shell Element in Geometric Nonlinear Analysis", Computers \& Structures, 2015, Vol. 146, pp. 91-104.

[45] Surana, K. S., "Geometrically Nonlinear Formulation for the Curved Shell Elements", International Journal for Numerical Methods in Engineering, 1983, Vol. 19, No. 4, pp. 581-615. 